\title{
Prefrontal White Matter Lesions and Prefrontal Task Impersistence in Depressed and Nondepressed Elders
}

\author{
Guy G Potter*,', Andrew D Blackwell', Douglas R McQuoid', Martha E Payne', David C Steffens', \\ Barbara J Sahakian², Kathleen A Welsh-Bohmer ${ }^{1,3}$ and KR Ranga Krishnan' \\ 'Department of Psychiatry and Behavioral Sciences, Duke University Medical Center, Durham, NC, USA; ${ }^{2}$ Department of Psychiatry, University of \\ Cambridge School of Clinical Medicine, Addenbrooke's Hospital \& Behavioural and Clinical Neuroscience Institute, University of Cambridge, \\ Cambridge, UK; ${ }^{3}$ Joseph and Kathleen Bryan Alzheimer's Research Center \& Division of Neurology, Duke University Medical Center, \\ Durham, NC, USA
}

\begin{abstract}
Poor task persistence is often observed among depressed individuals, and may be associated with some of the same frontal regions that are involved in depression. The current study explored the association between white-matter lesion volume in prefrontal cortex and noncompletion rates on a complex neurocognitive task among older adults in a treatment study for depression. Older adults in treatment for depression $(n=83)$ and nondepressed $(n=47)$ elders were administered the Stockings of Cambridge subtest (SoC) of the Cambridge Automated Neuropsychological Testing Battery (CANTAB) and completed a brain magnetic resonance imaging scan as part of an ongoing research study. Noncompletion of the SoC occurred in approximately 19\% of depressed participants (I 6/83) and only $2 \%$ of nondepressed participants (1/47), which was statistically significant. In multivariate models, failure to complete the SoC was consistently and significantly associated with greater volume of white matter lesions in the anterior-most region of prefrontal cortex, particularly in the left hemisphere, and with greater age. Although SoC completion was not significantly associated with depression severity, noncompletion rates were significantly higher among unremitted individuals and those with comorbid anxiety at study entry. The inability to initiate behavior sufficient to sustain a complex neurocognitive task is a characteristic of geriatric depression which may be associated with integrity of left-prefrontal regions. Future research should investigate whether task impersistence is a construct that generalizes to other neurocognitive tasks, and if it is associated with other adverse outcomes in geriatric depression related to cerebrovascular pathology, such as poor treatment response.
\end{abstract}

Neuropsychopharmacology (2007) 32, 2135-2 I42; doi: I0.1038/sj.npp. I 301339; published online I4 February 2007

Keywords: depression; elderly; prefrontal cortex; white matter lesions; neuropsychological testing; Stockings of Cambridge

\section{INTRODUCTION}

Geriatric depression is associated with impairment in executive functions that may be mediated through cerebrovascular pathology in subcortical structures and their projections to the frontal cortex (Alexopoulos et al, 2002b). One area of particular interest is deficits in self-initiated behavior, which have been associated with reduced treatment response (Alexopoulos et al, 2000; Kalayam and Alexopoulos, 1999) and higher levels of functional disability in depression (Kiosses et al, 2001). Multiple areas of the frontostriatal pathway are involved in the process of planning, initiating, and maintaining complex behavioral responses, including orbitofrontal cortex, dorsolateral

\footnotetext{
*Correspondence: Dr GG Potter, Department of Psychiatry and Behavioral Sciences, Duke University Medical Center, DUMC Box 3925, Durham, NC 277I0, USA, Tel: + I $9196826722 \times 250$, Fax: + I 919684 0424, E-mail: guy.potter@duke.edu

Received 30 March 2006; revised 18 December 2006; accepted 19 December 2006
}

prefrontal cortex, and anterior cingulate (Rogers et al, 1998). White matter lesions in these broader brain regions are more prominent in depressed older adults relative to nondepressed elders (Taylor et al, 2005), and like initiation deficits, are associated with greater risk for adverse outcomes in depression, such as poor treatment response (Alexopoulos et al, 2002a; Taylor et al, 2003) and functional impairment (Steffens et al, 2002a). Identifying neuropsychological performances that are sensitive to prefrontal vascular pathology would be useful for characterizing individuals with this form of late-life depression.

Although previous studies have identified initiation and perseveration as neuropsychological correlates of frontostriatal dysfunction in geriatric depression (Lockwood et al, 2002), less attention has been devoted to what is perhaps a more pathognomonic indicator, which is the failure to persist on tasks requiring these abilities. In behavioral terms, persistence is often defined as a function of how long an individual continues to initiate activity without receiving immediate reward (Pittenger, 2002). Deficits in reward-related responses have been associated with 
prefrontal cortex generally and specifically in depression (Naranjo et al, 2001; Reischies, 1999; Rolls, 2000), and persistence as a behavioral trait has also been linked to the prefrontal region (Gusnard et al, 2003). Although failure to persist on cognitive tasks has not been explicitly studied in the context of depression, factors that are likely to lead to this kind of performance deficit among depressed individuals - poor frustration tolerance, indecisiveness, lack of planning - were found to be prominent among individuals with frontal lobe lesions involving ventromedial prefrontal areas (Barrash et al, 2000). A study of frontotemporal dementia found that increasing illness severity was associated with a higher rate of incomplete tasks owing to stimulus-bound and perseverative behaviors, which was most prominent on tests of executive functions and reasoning (Smeding and de Koning, 2000). Applying these findings to geriatric depression, it is possible that the failure to persist on a cognitively demanding task is a diagnostically relevant occurrence that reflects dysfunction in prefrontal cortex, particularly the white matter pathways that are otherwise implicated in adverse outcomes in geriatric depression.

In this study, we examined the relationship between frontal white matter lesions on magnetic resonance imaging (MRI) and persistence on a complex cognitive task among depressed older adults and a nondepressed comparison group. We used a novel MRI processing methodology that quantified white matter lesion volumes in the anterior-most region of the brain. As a measure of task persistence, we examined completion rates on the Stockings of Cambridge subtest (SoC) of the Cambridge Automated Neuropsychological Testing Battery (CANTAB) (Cambridge Cognition Limited, 2004). This analog of the Tower of London (TOL) paradigm has been used as an index of prefrontal function in a variety of clinical populations, including patients with schizophrenia (Tyson et al, 2004), autism (Ozonoff et al, 2004), and in young (Purcell et al, 1997), and old adults with unipolar depression (Beats et al, 1996). In TOL-type tasks, colored disks or balls must be moved one-by-one from an initial pattern to a goal pattern that is presented to the participant. Participants are typically instructed to plan the entire sequence of moves mentally before executing it, which requires spatial working memory and organization. Previous research has shown that the anterior-most regions of prefrontal cortex are engaged on TOL-type tasks as planning demands increase (Baker et al, 1997), and that depressed individuals demonstrate less activation of prefrontal and frontostriatial areas relative to nondepressed individuals (Elliott et al, 1997). In the current study, we hypothesized that failure to persist to completion of the SoC task would be associated with depression status and greater extent of white matter lesions in the anterior-most regions of prefrontal cortex. In addition, we evaluated the relationship between clinically rated deficits in self-initiation behaviors and noncompletion of the SoC task.

\section{PARTICIPANTS AND METHODS}

\section{Sample}

Participants $(n=83)$ in the depressed group were enrolled in the National Institute of Mental Health-sponsored Conte
Center for the Neuroscience of Depression in Late-Life at Duke University Medical Center (DUMC). All participants diagnosed as depressed met DSM-IV criteria for major depression and were at least 60 years of age at baseline enrollment. The mean MADRS score at study entry was $24.85(\mathrm{SD}=6.67)$. At the time of assessment on the SoC, $70 \%$ of the depressed participants were under treatment with an antidepressant medication based on a standardized treatment algorithm (Steffens et al, 2002b). Among these participants, 37 were receiving monotherapy (27 on an SSRI, five on bupropion, two on nortriptyline, and one each on mirtazepine, methylphenidate and tranylcypromine); 21 were receiving combination treatment (11 on SSRI/bupropion, four on venlafaxine/bupropion, two on SSRI/mirtazepine, and one each on mirtazepine/bupropion, nortriptyline/SSRI, nortriptyline/bupropion, and venlafaxine/methylphenidate). The remaining 25 participants in the depressed group were receiving no antidepressants at the time of testing. The nondepressed control participants $(n=47)$ were screened for depression by the treating study geropsychiatrist at baseline and at yearly intervals using standardized clinical assessments. Exclusion criteria for both groups included (1) another major psychiatric illness, including bipolar disorder, schizophrenia, or dementia; (2) alcohol or drug abuse or dependence; (3) primary neurologic illness, including dementia; (4) medical illness, medication use, or disability that would prevent the participant from completing neurocognitive testing; and (5) contraindications to MRI. Depressed individuals meeting criteria for comorbid generalized anxiety disorder (GAD) were not excluded $(n=22)$. The complete description of study enrollment protocol is described elsewhere (Steffens et al, 2004). This study was approved by the Duke University Institutional Review Board. After receiving a complete description of the study, all participants provided written informed consent.

\section{SoC}

Participants who were enrolled in the study longitudinally were recruited to complete a brief battery of computerized tests aside from their yearly neuropsychological assessments. The Mini-Mental State Examination (MMSE, Folstein et al, 2001) was also administered. Many of these volunteers were under treatment and not required to be actively depressed. The SoC is the focus of this study because the task requires planning and executing a series of responses over numerous trials, which requires persistent self-initiation. The goal of the task is to move computerized balls to match a series of target patterns in the minimum number of moves and without breaking specified rules. The SoC has been found to be stable in healthy adults over a 9month re-test interval for minimum-move solutions and thinking time (Tyson et al, 2004). Factor analysis of the SoC found key indices to load on factors associated with spatial working memory and attentional shifting among healthy adults (Robbins et al, 1998). The SoC was administered on a desktop computer with a touchscreen monitor interface. Administration was conducted in accordance with the CANTAB administration manual (Cambridge Cognition Limited, 2004). 


\section{Depression Severity}

Participants in the depressed group were assessed for severity of depressive symptomatology at or near the time of their neuropsychological assessment (0.76 days, \pm 4.98$)$ based on the Montgomery-Asberg Depression Rating Scale (MADRS; Montgomery and Asberg, 1979). The MADRS is a 10 -item scale of depression severity that is based on patient report and clinical observation; in the current study, it was completed by the participant's treating psychiatrist. Scores on the MADRS range from 0 to 60 , with higher scores indicating greater severity of depressive symptomatology. Clinical ranges are typically considered to be 15-22 for mild depression, 22-34 for moderate depression, and $>35$ for severe depression (Muller et al, 2000). In the current study, we also defined remitted depression as a MADRS score less than 7. In addition to examining broad depressive symptomatology, we examined a single item on the MADRS reflecting deficits in initiation. This item has been conceptualized as assessing 'lassitude,' and has a scale reflecting 0 as the lowest level of signs and symptoms and 7 as the highest level of signs and symptoms. Higher scores on the MADRS Lassitude item were found to be associated with subcortical ischemic pathology in geriatric depression (Krishnan et al, 2004).

\section{MRI Variables}

MRI acquisition. Participants enrolled in the larger research protocol have completed brain MRI scans approximately every 2 years. In the current study, MRI data were obtained from the nearest MRI scanning date occurring before administration of the SoC (581.55 days, \pm 478$)$. All participants were screened for conditions that would preclude the use of MRI. Participants underwent imaging with a 1.5 -T whole-body MRI system (Signa, GE Medical Systems, Milwaukee, WI) using the standard head (volumetric) radiofrequency coil. The scanner alignment light was used to adjust the head tilt and rotation so that the axial plane lights passed across the cantho-meatal line, and the sagittal lights were aligned with the center of the nose. A rapid sagittal localizer scan was acquired to confirm the alignment.

A dual-echo, fast spin-echo acquisition was obtained in the axial plane for morphometry. The pulse sequence parameters were: repetition time, $4000 \mathrm{~ms}$; echo time, 30; $135 \mathrm{~ms}, 32 \mathrm{kHz}( \pm 16 \mathrm{kHz})$ full imaging bandwidth, echo train length $=16$, a $256 \times 256$ matrix, $3 \mathrm{~mm}$ section thickness, one excitation, and a $20 \mathrm{~cm}$ field of view. Images were obtained in two separate acquisitions. A 3-mm gap between sections was used in both acquisitions, with the second acquisition offset by $3 \mathrm{~mm}$ from the first so that the resulting data set consisted of contiguous sections.

MRI image processing. Images were processed in DUMC's Neuropsychiatric Imaging Research Laboratory (NIRL) on SUN workstations. Volume measurements were performed with a NIRL-modified version of MrX software, which was created by GE Corporate Research and Development (Schenectady, NY) and originally modified by Brigham and Women's Hospital for image segmentation (Boston, MA). The segmentation protocol and process for converting image intensity to segmented tissue types has been described previously (Payne et al, 2002). Briefly, it is a semi-automated method that uses the multiple MR contrasts available to identify different tissue classifications through a 'seeding' process, in which a trained analyst manually selects pixels in each tissue type to be identified (gray matter, white matter, cerebrospinal fluid, lesions or background). The seeding protocol identifies the range of signal intensities that characterize each tissue type. Lesion areas were selected based on a set of explicit rules that were developed from neuroanatomical guidelines, consultation with a neuroradiologist, and knowledge of the neuropathology of lesions (Payne et al, 2002). Periventricular lesions were defined as regions that were contiguous with the lateral ventricle and did not extend into the white matter tracts. They were classified as white matter lesions on the segmented image. Deep white matter lesions were located in the white matter tracts and may or may not have adjoined periventricular lesions.

Additional procedures were required to obtain measures of white matter lesion volume in the anterior or posterior halves of the brain. First, an axial plane was created along the anterior commissure-posterior commissure (AC-PC) line, dividing superior regions from inferior. Next coronal planes were created perpendicular to the axial plane at the anterior and posterior extent of the corpus callosum. Finally, a third coronal plane was created at the midpoint between the first two coronal planes, which divided the brain into anterior and posterior halves in each hemisphere.

In the current study, we examined white matter lesions in the area of the brain that was anterior to the genu of the corpus callosum. In addition, we examined the right and left hemispheres within this anterior-most section.

Training and reliability. All technicians received extensive training by experienced volumetric analysts. Reliability was established by repeated measurements on multiple MRI scans before raters were approved to process study data. Reliability estimates were based on two raters analyzing 10 scans, with raters analyzing each scan twice. Intraclass correlation coefficients were as follows: left cerebral white matter lesions, 0.988; right cerebral white matter lesions, 0.994 .

\section{Data Analysis}

The plan for data analysis included examination of bivariate and multivariate associations among depression, lesion volume, SoC completion, and relevant covariates. Bivariate analysis of SoC completion, lesion volumes and other relevant variables were examined using zero-order correlation, $t$-test, $\chi^{2}$, and Fisher's exact test as appropriate. Multivariate models were tested using logistic regression, in which the dependent variable was SoC completion, the primary independent variable was lesion volume, and the covariates were age, education, depression severity, GAD at study entry, and number of elapsed days between MRI and SoC. Lesion volume and the covariates were entered into the models simultaneously. Separate models were examined for lesion volume in whole AWM, left AWM, and right AWM. To examine better the specific effects of poor initiation on $\mathrm{SoC}$ completion, we generated a similar set of logistic 
regression models that replaced the total MADRS score reflecting depression severity with the single MADRS Lassitude item. Again, we examined separately models for lesion volume in whole AWM, left AWM, and right AWM. Finally, we ran the models substituting a proxy variable denoting remission status for what were previously the MADRS and Lassitude variables. Secondary to these models, we tested separately for interactions between lesion volumes and the variables of depression severity, Lassitude, and remission status.

\section{RESULTS}

The characteristics of the depressed and nondepressed groups are described in Table 1 . There were statistically significant differences between depressed and nondepressed participants with respect to age and education, but not sex. Cognitive status as assessed by the MMSE was not significantly different between depressed $(M=28.86$, $\mathrm{SD}=3.71)$ and nondepressed $(\mathrm{M}=29.38, \mathrm{SD}=1.51)$. Age at time of MRI scan was not significantly different between depressed and nondepressed. Lesion volumes in AWM (whole), left AWM, and right AWM were not significantly different between depressed and nondepressed groups. Among the depressed participants only, there was no statistically significant relationship between AWM lesion volumes and depression severity on the MADRS, and no significant association between lesion volumes and lassitude. AWM lesion volumes were also not significantly different between remitted and non-remitted participants, and there were no differences in lesion volumes between individuals with and without comorbid GAD at study entry.

Analyses of SoC completion rates between the two participant groups indicated that approximately $19 \%$ of depressed participants (16/83) did not complete the SoC, whereas approximately $2 \%$ of nondepressed participants did not complete this task $(1 / 47)$. This difference was statistically significant $\left(\chi^{2}=7.76, p<0.01\right)$. Individuals who did not complete the SoC were significantly older (Table 1). Among the depressed participants, the level of symptomatology on the MADRS was significantly lower $(t=2.38$, $p<0.05)$ for SoC completers $(M=6.84, S D=7.12)$ than for noncompleters $(M=11.69, S D=8.19)$. Individuals defined as unremitted depressed were significantly less likely to complete the SoC than individuals defined as remitted depressed $\left(\chi^{2}=8.76, p<0.01\right)$. There was no difference between completers and noncompleters in depression severity at entry to the study; however, noncompleters were significantly more likely to have met criteria for both depression and GAD at study entry (7/16, Fisher's exact $\leqslant 0.0474)$. Bivariate analysis indicated that AWM lesion volumes were larger among individuals who did not complete the SoC, but that this was statistically significant only for left AWM $(p<0.0431)$. We also examined this as a dichotomous variable based on whether individuals had any AWM lesions vs no measurable lesions. We found that approximately $71 \%$ of individuals who did not complete the SoC had at least some measurable white matter lesions in the anterior-most frontal region, whereas approximately $29 \%$ had no measurable lesions in this area $\left(\chi^{2}=4.11\right.$, $p=0.0426)$. These results were similar for both left$\left(\chi^{2}=5.69, p=0.0170\right)$ and right-sided lesions $\left(\chi^{2}=7.63\right.$, $p=0.0058)$.

Results from multivariate regression models showed a relatively consistent pattern of lesion-related effects when controlling for age, education, depression severity, GAD at study entry, and number of elapsed days between MRI and SoC. To analyze the effect of depression severity on SoC completion, we excluded the single nondepressed individual who failed to complete the SoC. Using a logistic model predicting completion status, we found greater lesion volume in AWM to be associated with a higher noncompletion rate on the SoC (Table 2), with age and GAD at study entry emerging as the other statistically significant predictors. When analyzing left AWM only, we again found that greater lesion volume, higher age, and GAD at study entry were associated with a higher rate of noncompletion

Table I Participant Characteristics, by Depression Status and SoC Completion Status

\begin{tabular}{|c|c|c|c|c|}
\hline & $\begin{array}{l}\text { Depressed } \\
\begin{array}{l}n=83 \\
M(S D)\end{array}\end{array}$ & $\begin{array}{l}\text { Nondepressed } \\
\qquad \begin{array}{l}n=47 \\
M(S D)\end{array}\end{array}$ & $\begin{array}{l}\text { SoC Complete } \\
\qquad \begin{array}{c}N=1 / 3 \\
M(S D)\end{array}\end{array}$ & $\begin{array}{c}\text { SoC incomplete } \\
n=17 \\
M \text { (SD) }\end{array}$ \\
\hline Age & $71.61(6.25)$ & $74.06(6.41)^{*}$ & $71.78(6.10)$ & 77.30 (6.38)*** \\
\hline Education & | $4.64(2.39)$ & I5.49 (1.6)* & I5.04 (2.07) & | $4.30(2.7 \mid)$ \\
\hline \multirow[t]{2}{*}{ \% Female } & 59 & 63 & 64 & 47 \\
\hline & $M(S E)$ & $M(S E)$ & $M$ (SE) & $M(S E)$ \\
\hline AWML & $0.094(0.026)$ & $0.0625(0.1859)$ & $0.0374(0.1308)$ & $0.3845(0.1732)$ \\
\hline Left AWML & $0.037(0.013)$ & $0.0307(0.1068)$ & $0.0179(0.07 \mid 8)$ & $0.1449(0.0575)^{* * * * *}$ \\
\hline Right AWML & $0.058(0.026)$ & $0.0318(0.0876)$ & $0.0195(0.0658)$ & $0.2396(0.1171)$ \\
\hline
\end{tabular}

$\mathrm{AWML}=$ anterior white matter lesion volume.

*Depressed vs nondepressed, $p<0.05$.

**Complete vs incomplete, $p<0.001$.

**** Complete vs incomplete, $p<0.05$. 
Table 2 Logistic Regression Predicting SoC Completion, Depressed Only $(n=83)$

\begin{tabular}{|c|c|c|c|}
\hline & $b$ & SE & $p$ \\
\hline AWML & -9.131 & 4.118 & 0.027 \\
\hline MADRS & -0.044 & $0.07 \mid$ & 0.542 \\
\hline GAD & -2.050 & 1.028 & 0.046 \\
\hline Education & 0.237 & 0.188 & 0.207 \\
\hline Age & -0.206 & 0.083 & 0.013 \\
\hline MRI date & -0.002 & 0.001 & 0.089 \\
\hline Left AWML & -26.238 & 11.322 & 0.025 \\
\hline MADRS & -0.035 & 0.072 & 0.664 \\
\hline GAD & -2.205 & 1.052 & 0.036 \\
\hline Education & 0.261 & 0.189 & 0.167 \\
\hline Age & -0.191 & 0.085 & 0.025 \\
\hline MRI date & -0.002 & 0.001 & 0.114 \\
\hline Right AWML & $-\mid 1.431$ & 6.772 & 0.09 \\
\hline MADRS & -0.033 & 0.068 & 0.629 \\
\hline GAD & -1.973 & 1.024 & 0.054 \\
\hline Education & 0.195 & 0.180 & 0.278 \\
\hline Age & -0.222 & 0.082 & 0.007 \\
\hline MRI date & -0.002 & 0.001 & 0.063 \\
\hline
\end{tabular}

$\mathrm{AWML}=$ anterior white matter lesion volume; $\mathrm{MADRS}=$ Montgomery-Asberg Depression Rating Scale; GAD = criteria for Generalized Anxiety Disorder at study entry; MRI date = days between MRI scan and SoC testing.

on the SoC. Analyzing right AWM alone, there was a significant effect of age, but no significant effects of lesion volume on failure to complete the SoC. There was no interaction between depression severity on the MADRS and any of the AWM variables. We then tested a similar logistic model predicting completion status with the lassitude item of the MADRS replacing the complete MADRS scale, and found a significant lesion effect of total AWM and left AWM (Table 3). Age was a significant predictor of SoC completion in the models, including total AWM, left AWM, and right AWM, but there was no significant effect of Lassitude or GAD. There was no interaction between lesion volume and Lassitude. Finally, because many of the individuals followed in our depressed cohort were in remission, we examined the same logistic model with a proxy variable denoting remission status. There was a significant effect of higher noncompletion among nonremitted individuals and for greater lesion volume in left AWM $(p<0.05$ for both variables). Age was also significantly associated with noncompletion, but there was no interaction between lesion volume and remission status.

\section{DISCUSSION}

The current study found that elderly individuals with either current or remitted depression were less likely than nondepressed elderly to persist to completion on a complex test of planning and organization. This failure to persist
Table 3 Logistic Regression Predicting SoC Completion, Controlling for MADRS 'Lassitude' Item

\begin{tabular}{|c|c|c|c|}
\hline & $b$ & SE & $p$ \\
\hline AWML & -9.215 & 4.269 & 0.008 \\
\hline Lassitude & -0.680 & 0.448 & 0.123 \\
\hline GAD & -1.707 & $1.00 \mid$ & 0.088 \\
\hline Education & 0.251 & 0.179 & 0.160 \\
\hline Age & -0.229 & 0.089 & 0.010 \\
\hline MRI date & -0.002 & 0.001 & 0.157 \\
\hline Left AWML & $-28.9 \mid 4$ & 12.245 & 0.018 \\
\hline Lassitude & -0.627 & 0.455 & 0.169 \\
\hline GAD & -1.810 & 1.022 & 0.077 \\
\hline Education & 0.267 & 0.179 & 0.137 \\
\hline Age & -0.203 & 0.088 & 0.022 \\
\hline MRI date & -0.002 & 0.001 & 0.150 \\
\hline Right AWML & $-|2.03|$ & 7.045 & 0.088 \\
\hline Lassitude & -0.707 & 0.440 & 0.109 \\
\hline Education & 0.233 & 0.176 & 0.185 \\
\hline GAD & -1.594 & 0.986 & 0.106 \\
\hline Age & -0.245 & 0.089 & 0.006 \\
\hline MRI date & -0.001 & 0.001 & 0.141 \\
\hline
\end{tabular}

$\mathrm{AWML}=$ anterior white matter lesion volume; $\mathrm{GD}=$ criteria for Generalized Anxiety Disorder at study entry; MRI date= days between MRI scan and SoC testing.

among depressed individuals was associated with higher volume of white matter lesions in anterior-prefrontal white matter, particularly in the left hemisphere. Although depression severity, clinically rated deficits in initiation (lassitude), and history of GAD at study entry each were significantly associated with higher rates of noncompletion in bivariate models, these three factors were unrelated to lesion volumes. Noncompletion was also associated with age, which is consistent with normative data on this task (Robbins et al, 1998). The current findings were based on an MRI-based method for localizing and quantifying lesion volume in prefrontal white matter.

The association between diminished integrity of prefrontal white matter and deficits in neuropsychological performance has been reported in other studies (Kramer-Ginsberg et al, 1999), and several studies have associated deficient performances on TOL-type tasks with compromises in prefrontal regions in both depressed (Goethals et al, 2005) and nondepressed populations (Newman et al, 2003). This study differed from prior research in that it focused on a neurobehavioral construct rather than on a specific level of neuropsychological performance. Impersistence was defined as a failure to maintain self-initiated responding and operationalized as failure to complete the SoC task, and this characteristic may be owing to a unique combination of neuroanatomical, neurocognitive, and behavioral deficits in any single individual. For instance, some depressed individuals may have a 'catastrophic' or abnormal response to task failure (Beats et al, 1996; Elliott et al, 2005), whereas 
others may have a deficient response to rewarding stimuli (Pizzagalli et al, 2005). The current research suggests that acquired cerebrovascular pathology in the anterior prefrontal region significantly raises the likelihood of task impersistence among depressed individuals even when not directly associated with specific symptoms or severity of depression. Although the current partitioning methodology does not identify specific prefrontal subregions that contribute to task impersistence on the SoC, research suggests that neurocognitive difficulties with TOL-type tests like the SoC in general may be related to multiple areas of the prefrontal cortex with white matter connections (Cazalis et al, 2003; Newman et al, 2003).

The stronger effect of left prefrontal lesions associated with task noncompletion is consistent with the original work on TOL-type tasks by Shallice (Shallice, 1982), which found deficits among individuals with left anterior lesions. The current research suggests that this left-sided effect may be a product of deficient plan execution mediated by left prefrontal regions. Previous study of TOL with functional MRI (Newman et al, 2003) found that the left prefrontal regions appeared to be more involved with plan execution, whereas the right prefrontal region was more involved with the generation of the plan. Other research has found that higher level performance on TOL ( $>70 \%$ correct) requires extended activation of left dorsolateral prefrontal cortex (Cazalis et al, 2003), and that increasing task complexity is associated with left anterior prefrontal regions (van den Heuvel et al, 2003). Further research needs to be conducted to understand how specific neurobehavioral deficits in depression are related to the laterality of white matter lesion burden.

There may be clinical utility to viewing task impersistence on other neurocognitive measures as a potential correlate of white matter pathology, greater deficits in initiation, decreased remission of depressive symptomatology, or other negative outcomes. Individuals who demonstrate a lack of persistence in a testing situation may be more likely to have problems with treatment adherence and other functional abilities. Like the task impersistence reported in the current study, poor treatment response and functional performance deficits have been found to be associated with white matter lesions at least partly involving the frontal lobes (Steffens et al, 2002a; Taylor et al, 2003). With additional research to clarify the construct, it is possible that task impersistence may be considered a marker for underlying prefrontal pathology and a higher risk of adverse clinical outcomes.

There are some limitations to the current study. One is that we do not have a complete explanation for why depressed and nondepressed individuals have similar lesion volumes in AWM, but the depressed have disproportionate difficulty completing the SoC, even while controlling for depression severity or remission status. Similarly, anxiety and lassitude were associated with higher rates of noncompletion on the SoC, but not with differences in lesion volumes. This highlights a difference from other research demonstrating relationships among age, depression, and prefrontal lesions (Taylor et al, 2005) and between subcortical ischemic pathology and lassitude (Krishnan et al, 2004). The absence of these expected relationships may reflect the fact that our MRI processing methodology focuses on lesion volume within a circumscribed brain region rather than on specific lesion sites. Also, by focusing on white matter in a relatively circumscribed area of the frontal lobes, we are not accounting for potential contributions to depression from more posterior structures of the frontostriatal system. There are also some areas of the prefrontal region that may not be included by our partitioning methodology, including posterior portions of the medial orbitofrontal and dorsolateral prefrontal regions. These broader regions may contribute more profoundly to other facets of depression that were not detected within our specific area of lesion focus. Thus, a low baseline level of lesions in the anterior AWM in conjunction with a relatively small sample of noncompleters may have resulted in sufficient power to detect the effects of AWM lesions on task impersistence, but insufficient power to detect differences between subgroups of our sample. Another potential explanation for the lack of group differences in lesion volumes comes from a study of white matter lesion volumes that did not find differences between depressed and nondepressed individuals when risk factors for cerebrovascular disease were excluded; however, they did find significantly more small lesions among depressed that were presumed to reflect infarcts or foci of edema, but no difference related to depression for larger lesions (Lenze et al, 1999). Although the circumscribed area we selected for this study precludes a detailed analysis of specific lesion types, we believe it likely that depressed and nondepressed individuals differed in a specific locus and type of lesion within the region that we assessed, and that this difference may account for higher likelihood of both depression and task impersistence.

We also acknowledge that the construct of task impersistence is not necessarily unitary, in that the etiology of noncompletion on the SoC may be owing to different relative influences of neurocognitive and neurobehavioral deficits for each specific individual. For example, although we demonstrated that greater lassitude and comorbid GAD at study entry were significant contributors to impersistence on the SoC, some individuals may have failed to complete the SoC because of greater cognitive difficulty than other participants. Post hoc analysis of MMSE differences between depressed completers and noncompleters was nonsignificant, which suggests that there was no gross discrepancy in cognitive status at the time of testing. Another potential influence is that some individuals may be less familiar with computerized testing than others; however, a cohort effect based on familiarity with computers would be unlikely given that completers and noncompleters were within 6 years of age on average and differed by less than 1 year of education. In the context of a naturalistic treatment design, there is potential for medication-specific treatment effects on neurocognitive performance due to the nonuniform use of psychotropic agents. In this study, however, there was no significant difference in SoC performance based on whether individuals were on psychotropic medication or not at the time of testing. Among those on medication, only four were on nortriptyline, and the remainder were on medications that generally have not been found to have an adverse effect on neurocognitive performance. Although there are advantages to single-agent studies of cognition, a naturalistic 
treatment design may have better ecological validity, and we do not have evidence that this methodology confounded our test findings. Finally, our assessment of comorbid anxiety was not ideal because it was assessed at study entry rather than at the time of testing; however, this was a relevant covariate despite the absence of a direct assessment of anxiety at the time of SoC administration. With respect to these limitations, we note that although task impersistence is likely multifactorial and heterogeneous across study participants, each individual must still make reward-reinforcement decisions based on their specific circumstances, and depressed individuals with greater lesion burden in AWM appeared to find less reward in maintaining their responses on the SoC than individuals with less lesion burden, above and beyond the influences of depression, anxiety, and general deficits in initiation.

Another limitation is that our MRI data were not uniformly related in time to the administration of the SoC, though all MRI scans occurred before the SoC and the range of days elapsed between these two assessments was nonsignificant as a covariate. Furthermore, depressed and nondepressed were comparable in age at the time they received their scans, suggesting no bias on this timedependent variable. It is possible that some participants may have developed additional prefrontal or other lesions over time, and participants who were depressed at the time of MRI were often less depressed at the time of SoC administration; however, previous research has shown that the pattern of change in white matter lesion volumes over 2 - and 4-year intervals is not significantly different between depressed and nondepressed individuals (Chen et al, 2006). In fact, any bias that may exist owing to lower lesion burden at time of MRI relative to subsequent SoC testing would presumably make it less likely to detect a significant effect of lesion volume on noncompletion. Although we did not have ideal timing intervals between MRI and SoC, we believe that our findings would actually be more robust with optimal timing intervals.

In summary, we believe that the inability to persist to completion of a complex task, whether owing to lesion pathology, depressive symptomatology- or both- may be an informative outcome in depression that is a marker for underlying cerebrovascular brain pathology in anterior prefrontal cortex. Future research should consider this form of 'missing data' on other neuropsychological tests, and whether impersistence is a construct that generalizes to other outcomes in geriatric depression, such as treatment response.

\section{ACKNOWLEDGEMENTS}

This study was supported in part by the Conte Center for the Study of Depression in Late-Life, including NIMH grants P50 (MH60451) and R01 (MH54846). Authors ADB and BJS were funded by the Wellcome Trust and worked within an MRC Center for Behavioral and Clinical Neuroscience when the manuscript was originally submitted. BJS is a consultant for Cambridge Cognition, and ADB assumed the position of Scientific Director for Cambridge Cognition during the revision of this manuscript.

\section{REFERENCES}

Alexopoulos GS, Kiosses DN, Choi SJ, Murphy CF, Lim KO (2002a). Frontal white matter microstructure and treatment response of late-life depression: a preliminary study. $A m \mathrm{~J}$ Psychiatry 159: 1929-1932.

Alexopoulos GS, Kiosses DN, Klimstra S, Kalayam B, Bruce ML (2002b). Clinical presentation of the 'depression-executive dysfunction syndrome' of late life. Am J Geriatr Psychiatry 10: 98-106.

Alexopoulos GS, Meyers BS, Young RC, Kalayam B, Kakuma T, Gabrielle $M$ et al (2000). Executive dysfunction and longterm outcomes of geriatric depression. Arch Gen Psychiatry 57: 285-290.

Baker SC, Rogers RD, Owen AM, Frith CD, Dolan RJ, Frackowiak RS et al (1997). Neural systems engaged by planning: a pet study of the Tower of London task. Neuropsychologia 34: 515-526.

Barrash J, Tranel D, Anderson SW (2000). Acquired personality disturbances associated with bilateral damage to the ventromedial prefrontal region. Dev Neuropsychol 26: 125-140.

Beats BC, Sahakian BJ, Levy R (1996). Cognitive performance in tests sensitive to frontal lobe dysfunction in the elderly depressed. Psychol Med 26: 591-603.

Cambridge Cognition Limited (2004). CANTABeclipse Test Administration Guide. Cambridge Cognition Limited: Cambridge, UK.

Cazalis F, Valabregue R, Pelegrini-Issac M, Asloun S, Robbins TW, Granon S (2003). Individual differences in prefrontal cortical activation on the Tower of London planning task: Implication for effortful processing. Eur J Neurosci 17: 2219-2225.

Chen PS, McQuoid DR, Payne ME, Steffens DC (2006). White matter and subcortical gray matter lesion volume changes and late-life depression outcome: a 4-year magnetic resonance imaging study. Int Psychogeriatr 18: 445-456.

Elliott R, Baker SC, Rogers RD, O'Leary DA, Paykel ES, Frith CD et al (1997). Prefrontal dysfunction in depressed patients performing a complex planning task: a study using positron emission tomography. Psychol Med 27: 931-942.

Elliott R, Sahakian BJ, Herrod JJ, Robbins TW, Paykel ES (2005). Abnormal response to negative feedback in unipolar depression: Evidence for a diagnosis specific impairment. I Neurol Neurosurg Psychiatry 63: 74-82.

Folstein M, Folstein S, Fanjiang G (2001). Mini-Mental State Examination: Clinical Guide and User's Guide. Psychological Assessment Resources: Lutz, FL.

Goethals I, Audenaert K, Jacobs F, Van de Wiele C, Ham H, Pyck H et al (2005). Blunted prefrontal perfusion in depressed patients performing the Tower of London task. Psychiatry Res 139: 31-40.

Gusnard DA, Ollinger JM, Shulman GL, Cloninger CR, Price JL, Van Essen DC et al (2003). Persistence and brain circuitry. Proc Natl Acad Sci USA 100: 3479-3484.

Kalayam B, Alexopoulos GS (1999). Prefrontal dysfunction and treatment response in geriatric depression. Arch Gen Psychiatry 56: 713-718.

Kiosses DN, Klimstra S, Murphy C, Alexopoulos GS (2001). Executive dysfunction and disability in elderly patients with major depression. Am J Geriatr Psychiatr 9: 369-374.

Kramer-Ginsberg E, Greenwald BS, Krishnan KRR, Christiansen B, $\mathrm{Hu}$ J, Ashtari M et al (1999). Neuropsychological functioning and MRI signal hyperintensities in geriatric depression. $A m J$ Psychiatry 156: 438-444.

Krishnan KR, Taylor WD, McQuoid DR, MacFall JR, Payne ME, Provenzale JM et al (2004). Clinical characteristics of magnetic resonance imaging-defined subcortical ischemic depression. Biol Psychiatry 55: 390-397.

Lenze E, Cross D, McKeel D, Neuman RJ, Sheline YI (1999). White matter hyperintensities and gray matter lesions in physically healthy depressed subjects. Am J Psychiatry 156: 1602-1607. 
Lockwood KA, Alexopoulos GS, van Gorp WG (2002). Executive dysfunction in geriatric depression. Am J Psychiatry 159: 1119-1126.

Montgomery SA, Asberg M (1979). A new depression scale designed to be sensitive to change. Br J Psychiatry 134: 382-389.

Muller MJ, Szegedi A, Wetzel H, Benkert O (2000). Moderate and severe depression. Gradations for the Montgomery-Asberg Depression Rating Scale. J Affect Disord 60: 137-140.

Naranjo CA, Tremblay LK, Busto UE (2001). The role of the brain reward system in depression. Prog Neuropsychopharmacol Biol Psychiatry 25: 781-823.

Newman SD, Carpenter PA, Varma S, Just MA (2003). Frontal and parietal participation in problem solving in the Tower of London: fMRI and computational modeling of planning and high-level perception. Neuropsychologia 41: 1668-1682.

Ozonoff S, Cook I, Coon H, Dawson G, Joseph RM, Klin A et al (2004). Performance on Cambridge neuropsychological test automated battery subtests sensitive to frontal lobe function in people with autistic disorder: evidence from the collaborative programs of excellence in autism network. J Autism Dev Disord 34: 139-150.

Payne ME, Fetzer DL, MacFall JR, Provenzale JM, Byrum CE, Krishnan KR (2002). Development of a semi-automated method for quantification of MRI gray and white matter lesions in geriatric subjects. Psychiatry Res 115: 63-77.

Pittenger DJ (2002). The two paradigms of persistence. Genet Soc Gen Psychol Monogr 128: 237-268.

Pizzagalli DA, Jahn AL, O'Shea JP (2005). Toward an objective characterization of an anhedonic phenotype: A signal-detection approach. Biol Psychiatry 57: 319-327.

Purcell R, Maruff P, Kyrios M, Pantelis C (1997). Neuropsychological function in young patients with unipolar major depression. Psychol Med 27: 1277-1785.

Reischies FM (1999). Pattern of disturbance of different ventral frontal functions in organic depression. Ann NY Acad Sci 877: 775-780.

Robbins TW, James M, Owen AM, Sahakian BJ, Lawrence AD, McInnes L et al (1998). A study of performance on tests from the CANTAB battery sensitive to frontal lobe dysfunction in a large sample of normal volunteers: Implications for theories of executive functioning and cognitive aging. J Int Neuropsychol Soc 4: 474-490.

Rogers MA, Bradshaw JL, Pantelis C, Philips JG (1998). Frontostriatal deficits in unipolar major depression. Brain Res Bull 47: 297-310.

Rolls ET (2000). The orbitofrontal cortex and reward. Cereb Cortex 10: $284-294$.

Shallice T (1982). Specific impairments of planning. Philos Trans $R$ Soc Lond B Biol Sci 25: 199-209.

Smeding HM, de Koning I (2000). Frontotemporal dementia and neuropsychology: the value of missing values. I Neurol Neurosurg Psychiatry 68: 726-730.

Steffens DC, Bosworth HB, Provenzale JM, MacFall JR (2002a). Subcortical white matter lesions and functional impairment in geriatric depression. Depress Anxiety 15: 23-28.

Steffens DC, McQuoid DR, Krishnan KRR (2002b). The Duke somatic treatment algorithm for geriatric depression (STAGED) approach. Psychopharmacol Bull 36: 58-68.

Steffens DC, Welsh-Bohmer KA, Burke JR, Plassman BL, Beyer JL, Gersing KR et al (2004). Methodology and preliminary results from the neurocognitive outcomes of depression in the elderly study. J Geriatr Psychiatry Neurol 17: 202-211.

Taylor WD, MacFall JR, Payne ME, McQuoid DR, Steffens DC, Provenzale JM et al (2005). Greater MRI lesion volumes in elderly depressed subjects than in control subjects. Psychiatry Res 139: 1-7.

Taylor WD, Steffens DC, MacFall JR, McQuoid DR, Payne ME, Provenzale JM et al (2003). White matter hyperintensity progression and late-life depression outcomes. Arch Gen Psychiatry 60: 1090-1096.

Tyson PJ, Laws KR, Roberts KH, Mortimer AM (2004). Stability of set-shifting and planning abilities in patients with schizophrenia. Psychiatry Res 129: 593-611.

van den Heuvel OA, Groenewegen HJ, Barkhof F, Lazeron RH, van Dyck R, Veltman DJ (2003). Frontostriatal system in planning complexity: a parametric functional magnetic resonance version of Tower of London task. Neuroimage 18: 367-374. 